\title{
Comparando dos estrategias de aprendizaje activo para enseñar Scrum en un curso introductorio de ingeniería de software
}

\author{
Comparing two active learning strategies for teaching Scrum in \\ an introductory software engineering course
}

\author{
Silvia I. Lozano ${ }^{1 *} \quad$ Elizabeth Suescún $^{1} \quad$ Paola Vallejo $^{1}$ \\ Raúl Mazo ${ }^{1,2} \quad$ Daniel Correa $^{1}$ \\ Recibido 7 de noviembre de 2017, aceptado 8 de octubre de 2018 \\ Received: November 7, 2017 Accepted: October 08, 2018
}

\begin{abstract}
RESUMEN
El aprendizaje activo consiste en cualquier método de instrucción que comprometa a los estudiantes en su proceso de aprendizaje a través de actividades o discusiones realizadas principalmente durante las clases. En este artículo se reportaron los resultados de la comparación de dos estrategias de aprendizaje activo para la enseñanza del marco de trabajo ágil Scrum en el contexto de un curso introductorio de Ingeniería de Software. La comparación se hizo a través de un cuasiexperimento en el que los participantes se dividieron en dos grupos. En un grupo se utilizó la estrategia de lectura activa sobre conceptos básicos de Scrum, mientras que en el otro grupo se utilizó un juego; dos técnicas usadas en el aprendizaje activo. Los resultados dieron indicios a nivel de población que hay diferencias significativas en cuanto a los conceptos aprendidos por los integrantes de los grupos y confirmaron el valor que tiene utilizar estrategias de aprendizaje activo para enseñar Scrum. En particular, los resultados aportaron evidencia empírica que indica que utilizar diversas estrategias de aprendizaje activo facilita la retención y apropiación de conceptos relacionados con Scrum, y los resultados constituyen un punto de referencia para los docentes sobre la efectividad de estos dos métodos de aprendizaje activo en la enseñanza de este marco de trabajo ágil.
\end{abstract}

Palabras clave: Scrum, aprendizaje activo, Ingeniería de software.

\begin{abstract}
Active learning comprises any process in which students are actively engaged in building understanding of facts, ideas, and skills through instructor-directed tasks and activities undertaken mainly during classes. In this paper we presented results from a comparison of two active learning strategies to teach the agile Scrum framework in the context of an introductory software engineering course. The comparison was carried out through a quasi-experiment in which participants were divided into two groups. The first group used the strategy of active reading on basic concepts of Scrum, while for the other group a game was used; representing two teaching strategies for active learning. The results gave indications at the population level that there are significant differences in the concepts learned by the members of both groups and ratifies the use of active learning strategies to teach Scrum. The results provided empirical evidence indicating that using various active learning strategies facilitates the retention and appropriation of concepts related to Scrum and offer teachers a point of reference about the effectiveness of these two strategies of active learning to teach Scrum concepts.
\end{abstract}

Keywords: Scrum, active learning, software engineering.

\footnotetext{
1 GIDITIC. Departamento de Informática y Sistemas. Universidad EAFIT. Carrera 49 No 7 Sur - 50. Medellín, Colombia. E-mail: slozanoa@eafit.edu.co, esuescu1@eafit.edu.co, pvallej3@eafit.edu.co, rimazop@eafit.edu.co; dcorreab@eafit.edu.co

2 Lab-STICC, ENSTA Bretagne. 2 rue François Verny, Brest - France. E-mail: raul.mazo@ensta-bretagne.fr

* Autor de correspondencia: slozanoa@eafit.edu.co
} 


\section{INTRODUCCIÓN}

Las tendencias para transformar la enseñanza evidencian cómo las estrategias de aprendizaje activo se han ido adoptando en la academia gracias a los buenos resultados obtenidos con dichas estrategias. Investigaciones en este campo muestran que los estudiantes aprenden y se desempeñan mejor si el ambiente de aprendizaje incluye actividades en las que ellos participan y aplican el conocimiento, en lugar de ser pasivos en el proceso y simplemente escuchar al profesor [1-2]. Así, por ejemplo, el enfoque de aprendizaje activo puede ser guiado por el profesor en el uso de estrategias como aprendizaje en grupo, aprendizaje basado en problemas, aprendizaje basado en proyectos, aula invertida, discusiones y hasta actividades lúdicas que generan debates $[1,3]$.

Varios estudios soportan los beneficios de las estrategias de aprendizaje activo en comparación con la instrucción tradicional $[1,2,4]$. Sin embargo, pocas investigaciones se han centrado en comparar los efectos diferenciales entre varias estrategias de aprendizaje activo. De acuerdo con Freeman en [2] sería más productivo enfocarse en investigaciones que comparen estrategias de aprendizaje activo entre sí para identificar cuál estrategia es más apropiada y eficiente para ciertos temas o tipos de estudiantes, en vez de solamente realizar comparaciones con la instrucción tradicional.

Lograr que el estudiante se comprometa activamente en lo que está tratando de aprender, es de forma general el enfoque del aprendizaje activo. Cada estrategia que se describe en este estudio, es decir, la actividad con el juego SimulES y la actividad de la lectura activa, representan una transferencia de responsabilidad del profesor al estudiante y las dos están encaminadas a lograr el mismo objetivo por caminos diferentes; además la comparación de la efectividad entre estos dos enfoques no ha sido abordada de manera clara en estudios previos. Es por eso por lo que se decide hacer un estudio comparativo de la efectividad de las dos estrategias de aprendizaje activo antes mencionadas en el contexto del marco de trabajo ágil de software llamado Scrum y los resultados son reportados en este artículo.

Este estudio pretende responder a la siguiente pregunta de investigación:
¿En qué medida una de dos estrategias de aprendizaje activo (el juego y la lectura activa) es más efectiva que la otra, para promover la enseñanza-aprendizaje de Scrum en estudiantes universitarios de primer semestre? Por efectiva, entendemos que la estrategia se relaciona con un resultado esperado al realizar las pruebas que miden el nivel de conocimiento del estudiante sobre los conceptos de Scrum, este nivel de conocimiento según la taxonomía de Bloom [5] se relaciona con la retención de la información.

En este estudio se identifican también los puntos de vista de los estudiantes con respecto al uso de las dos estrategias de aprendizaje activo y su satisfacción en relación con dichas estrategias.

El análisis comparativo nos permitió encontrar que los estudiantes que participaron en la lectura activa en clase mostraron un mejor resultado en el conocimiento adquirido sobre los conceptos de Scrum en comparación con los estudiantes que usaron el juego SimulES.

El resto del documento se estructura como sigue: en la segunda sección se presentan los conceptos básicos sobre aprendizaje activo. En la tercera sección se describen algunos trabajos previos relacionados con el problema. La cuarta sección expone la metodología utilizada para llevar a cabo el estudio. Posteriormente, en la quinta sección se exponen los resultados y las amenazas a la validez presentes en el estudio. Finalmente, en la sexta sección se presentan las conclusiones y un panorama de posibles trabajos futuros en el tema del artículo.

\section{MARCO TEÓRICO}

El aprendizaje activo es una estrategia que ha recibido una considerable atención por la evidencia asociada a resultados positivos en el desempeño de los estudiantes cuando es aplicada en clase $[2,4,37]$. El aprendizaje activo, en relación con estrategias tradicionales, en las que el estudiante es pasivo en su proceso, resulta atractivo porque se centra en el aprendizaje del estudiante a través de una experiencia de colaboración y de reflexión individual y continua [3]. En otras palabras, en la estrategia de aprendizaje activo el estudiante es motivado a desarrollar su conocimiento y habilidades a través de cuestionamientos, búsquedas, análisis 
y síntesis de información, así como la adopción de una postura activa para solucionar problemas.

Prince [1] indaga sobre las evidencias relacionadas con la efectividad de esta estrategia y pretende consolidar su revisión en estudios de aprendizaje activo que sean relevantes en la enseñanza de la ingeniería. Según Prince, el aprendizaje activo crea en los estudiantes de ingeniería un cambio de comportamiento dado por una experiencia de aprendizaje que lo obligan a hacer y a reflexionar sobre lo que está haciendo. Es importante resaltar que el aprendizaje activo debe involucrar actividades que son aplicadas en clase y de las cuales se debe evidenciar lo aprendido. Es en este aspecto que se contrasta con las clases magistrales, donde el estudiante recibe pasivamente la información del profesor o instructor, para lo cual se puede correr el riesgo de que la misma no se utilice y por tanto, el cerebro puede prescindir de ella.

En este mismo contexto, King [6] afirma que cuando los estudiantes están comprometidos o involucrados activamente en procesar información (reconstrucción, por ejemplo) hace que ellos probablemente recuerden mejor lo aprendido y puedan aplicar esos conocimientos a nuevas situaciones. Dicha afirmación es sustentada por la teoría sobre procesamiento de información expuesta por Mayer [7], quien argumenta que la reformulación genera nueva información, ya que se construyen estructuras cognitivas que conectan las nuevas ideas, enlazando éstas con las conocidas previamente. A diferencia de los métodos tradicionales, el aprendizaje activo está centrado en el estudiante quien participa pensando y discutiendo ideas para hacerlas significativas, y en palabras del autor, el profesor ya no es esa figura de "sabio en el escenario, sino, un guía que está al lado". Adicional, el profesor posibilita el aprendizaje, pero es menos directo, más bien es responsable de guiar y presentar el material de la clase o actividad, necesarias para la interacción y manipulación de ideas.

Para entender las estrategias de aprendizaje activo es importante entender cómo los estudiantes aprenden. El trabajo de Wankat y Oreovicz [8] presenta cuestionamientos en este aspecto, principalmente en el "qué" deberían hacer los profesores para ayudar en el aprendizaje de los alumnos y por qué los diferentes métodos de aprendizaje producen diferentes efectos en ellos.
A pesar de años de investigación todavía no existe una respuesta conclusiva, sin embargo, las evidencias son útiles para que los profesores entiendan sobre el proceso de enseñanza-aprendizaje, conociendo que los diferentes estilos de aprendizaje deben ser considerados para planear cursos y manejar las relaciones interpersonales. La esencia de lo expuesto por estos autores tiene como base el trabajo de Myers y McCauley [9] y la teoría de Jung [10]. Se afirma que hay una variación aparentemente aleatoria relacionada al comportamiento, sin embargo, dicha aleatoriedad no existe, el comportamiento es ordenado y consistente, todo eso se debe a las diferencias básicas en la manera en que los individuos usan la percepción y el juicio. Percepción como aquella manera en la que se procesa la información y se es consciente del mundo alrededor de la persona y el juicio como la manera en que se toman decisiones sobre lo percibido. Cabe resaltar, que los autores afirman que se puede descubrir la forma preferida de tratar y vivir en el mundo por parte de las personas. Las preferencias intrínsecas, aunque posiblemente innatas, no siempre están disponibles en las mentes conscientes de las personas. La forma en que son educadas y las situaciones a las que se enfrentan pueden obligarlas a reaccionar de manera opuesta a sus preferencias inherentes. El aprendizaje activo podría abordar de forma más amplia la diversidad en los procesos de aprendizaje.

En la práctica, de acuerdo con lo expresado por Prince [1] el aprendizaje activo se refiere a las actividades que se introducen y realizan en el aula. De lo cual se afirma que los elementos básicos del aprendizaje activo son la actividad del estudiante y su participación en el proceso de aprendizaje. El autor resalta que los métodos instruccionales del aprendizaje activo a menudo se contrastan con estrategias tradicionales en la que los estudiantes reciben pasivamente información del profesor o instructor. Algunas de las actividades susceptibles de relacionar son: aprendizaje colaborativo, aprendizaje cooperativo, aprendizaje basado en problemas y otras mencionadas con anterioridad. Aquí se resalta la importancia de gestionar de forma pertinente actividades grupales y en lo que respecta a convertir grupos de estudiantes en equipos eficaces, Oakley [11] presenta una serie de sugerencias relacionadas a los beneficios del trabajo colaborativo y en grupo. Los resultados son mejores cuando los estudiantes aprenden en grupos pequeños que aquellos que 
aprenden de forma tradicional. El aprendizaje en grupo permite retener la información por más tiempo, menor probabilidad de abandonar los objetivos, se adquieren habilidades de comunicación y trabajo en equipo y se gana en una mejor comprensión del entorno en el cual se trabaja.

Otras estrategias de aprendizaje activo incluyen: juegos, simulaciones, juegos de roles, casos, casos cooperativos, debates, discusiones, enseñanza en pares y mapas de conceptos [12].

De acuerdo con Vlachopoulos en [36] los juegos son herramientas pedagógicas y artificiales que incluyen conflicto, reglas específicas y objetivos predeterminados que deben ser seguidos por los participantes mientras que la lectura activa es una estrategia donde el estudiante proactivamente se involucra en la lectura de un texto y se apoya en prácticas tales como resaltar, leer y pensar en voz alta, hacer predicciones, cuestionar y debatir en pequeños grupos sobre un tema específico [13].

\section{TRABAJOS RELACIONADOS}

\section{Estrategias para el aprendizaje de Scrum}

Tradicionalmente se han usado diferentes modelos para el ciclo de vida del desarrollo de software, por ejemplo, cascada [14-16], espiral [17] y modelo V [18-19]. Sin embargo, los métodos ágiles están siendo cada vez más adoptados por la industria del software mundial; por ejemplo, el 14\% de las compañías de software en Estados Unidos y Europa dijeron que los utilizaban y el $49 \%$ manifestaron interés en utilizarlos [20].

Scrum es uno de los marcos de trabajo ágiles más recientes y es usado en diversas compañías con el fin de producir software en corto tiempo y de reducir los costos de producción sin tener que sacrificar las exigentes solicitudes de los clientes [21]. Cardozo [20] presenta una revisión de literatura que evidencia la correlación entre el uso de Scrum y la mejora de la productividad en los proyectos de software; en la revisión se muestran evidencias positivas sobre la satisfacción de los clientes, la calidad del proceso y del producto, la motivación del equipo y la reducción de costos. En Scrum cada miembro del equipo (Product owner, Scrum master, Scrum team) trabaja en conjunto con los demás para entregar el proyecto en el menor tiempo posible con costos mínimos. Scrum está basado en la flexibilidad, la adaptabilidad, la creatividad y la productividad; además permite identificar y monitorear problemas en tiempo real durante el proceso de desarrollo del proyecto. Estas características han llevado a que sea uno de los métodos más populares en el contexto del desarrollo ágil de software.

Para responder a las necesidades de las compañías en la gestión de los equipos de desarrollo, es importante enseñar estrategias como los métodos ágiles dentro de los cursos de Ingeniería de Software [22]. Los educadores se enfrentan entonces al interrogante de cómo incorporar métodos ágiles como Scrum en sus cursos. Mahnic [22] presenta una revisión de literatura relacionada con la enseñanza de Scrum en cursos de Ingeniería de Software en la cual encuentra que la enseñanza de este marco de trabajo ágil no se limita a sesiones magistrales, sino que se complementa con actividades prácticas debido a que este concepto "es fácil de entender, pero difícil de dominar" [23]. Según ese estudio de la literatura, algunas de las actividades usadas para la enseñanza de Scrum en entornos académicos son: trabajo práctico a través de proyectos en equipos, juegos educativos y juegos de simulación. Además, Mahnic [22] indica que la manera más extendida para enseñar Scrum es a través de trabajos prácticos (proyectos de software) realizados por los estudiantes, permitiéndoles reemplazar las largas sesiones de clases teóricas/magistrales por sesiones prácticas. Con respecto a los juegos educativos, se indica que son particularmente adecuados cuando no se cuenta con suficiente tiempo para desarrollar proyectos complejos porque los estudiantes siguen las reglas y prácticas de Scrum mientras desarrollan un producto simple.

En la literatura se encuentran diferentes juegos. Por ejemplo, Paasivaara [24] propone que los estudiantes usen LEGO ${ }^{\circledR}$ con el fin de adquirir los objetivos de aprendizaje relativos al proceso de Scrum, la gestión de requisitos y la colaboración con los clientes, la estimación, el trabajo en equipo y la visualización del progreso. Otro ejemplo del uso de juegos en el aprendizaje de Scrum fue el uso de PlayScrum (un juego de cartas para el aprendizaje de Scrum) [25] que tiene como objetivo enseñar lecciones del mundo real que serían difíciles de aprender con otros enfoques de aprendizaje tradicional. Como complemento de esos trabajos, en este artículo se 
reportan los resultados de una comparación entre dos estrategias de aprendizaje activo para enseñar Scrum: (i) el uso del juego SimulES, y (ii) la lectura activa sobre conceptos básicos de Scrum, con el objetivo de identificar evidencias preliminares sobre la efectividad de cada estrategia para facilitar el conocimiento y retención de los conceptos básicos de Scrum.

\section{SimulES - Scrum}

SimulES es un juego educativo [26] que surgió como evolución del juego de cartas Problems and Programmers (PnP) [27]. SimulES permite a los jugadores explorar pedagógicamente el proceso de desarrollo de software y a su vez adquirir conocimientos de los conceptos básicos de Ingeniería de Software, al no contar con un proceso de desarrollo especificado, los jugadores cuentan con la libertad de adoptar diversas estrategias, mientras un jugador usa un enfoque de cascada, otro podría estar usando un enfoque ágil. Los componentes principales del juego son las cartas: Proyecto, Equipo de desarrollo, Concepto y Problema. En la Figura 1 se observa un ejemplo de dichas cartas adaptadas para el contexto de Scrum.
Las cartas Proyecto incluyen una descripción del proyecto que se debe desarrollar y contienen la lista de historias de usuario que se deben presentar como resultado de la implementación del proyecto. Las cartas Equipo de desarrollo indican una descripción de los diferentes roles que pueden ser contratados para participar en el proyecto de acuerdo con Scrum. Las cartas Concepto presentan los conceptos importantes sobre el método de trabajo Scrum; y las cartas Problema presentan situaciones o condiciones que se pueden presentar durante el desarrollo del proyecto y que pueden entorpecer su realización en un contexto ágil.

SimulES ha sido utilizado en experiencias previas para la enseñanza de conceptos básicos de Ingeniería de Software a estudiantes de Ingeniería informática [28, 29, 30, 31]. Para el propósito de este artículo el juego SimulES fue adaptado, desde su contenido y desde la dinámica misma del juego con el fin de orientarlo a la enseñanza de Scrum. Los cambios principales que se realizaron fueron los siguientes: 1) las cartas de los Ingenieros de Software se convirtieron en cartas de Equipo de desarrollo con los tres roles principales de Scrum; 2) el contenido de las

Fuente: los autores.

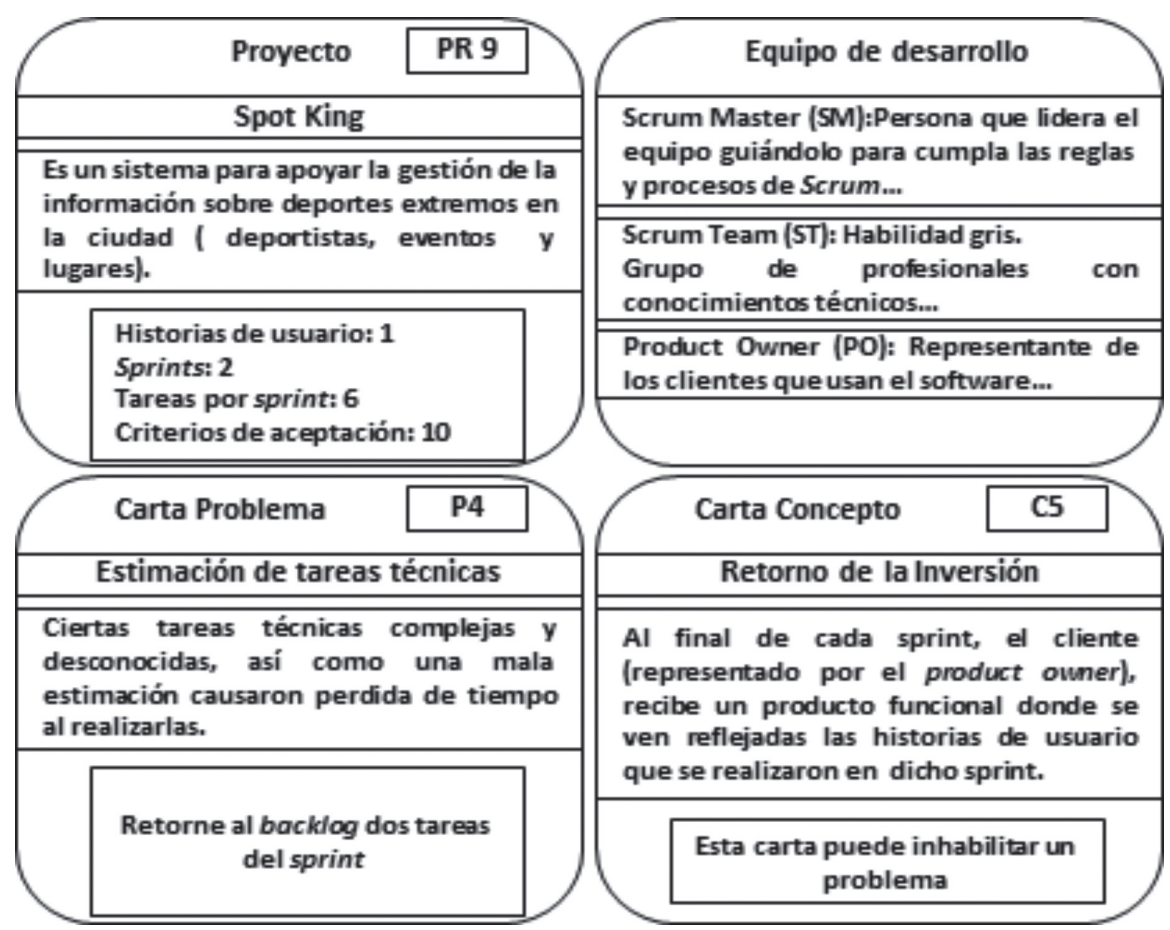

Figura 1. Cartas del juego SimulES usando conceptos Scrum. 
cartas Concepto y de las cartas Problema se modificó para incluir elementos y escenarios asociados al contexto ágil y a Scrum; y 3) la descripción de las cartas Proyecto también fue especializada para proyectos desarrollados con Scrum.

\section{METODOLOGÍA DE INVESTIGACIÓN}

Durante los últimos años, científicos e industriales se han servido de los estudios empíricos con estudiantes para evaluar hipótesis, para obtener evidencia empírica acerca del uso de nuevas propuestas y para responder a preguntas de investigación mediante experimentos controlados, cuasiexperimentos, estudios de correlación y encuestas $[32,33]$.

Dadas las características del estudio reportado en este artículo, y que los grupos de estudiantes participantes del estudio no podían ser asignados aleatoriamente, se decidió desarrollar un cuasiexperimento con el fin de estimar el impacto causal entre el uso de dos técnicas de aprendizaje activo y la efectividad de cada técnica en el aprendizaje de Scrum. Además, es importante resaltar que la asignación al azar no siempre es deseable o posible en estudios relacionados con la Ingeniería de Software puesto que, por ejemplo, las habilidades y el conocimiento previos son determinantes en el proceso de desarrollo de software y la asignación aleatoria puede conducir a grupos desbalanceados en esos aspectos [34].

El diseño del cuasiexperimento incluyó dos condiciones: un grupo experimental 1 de comparación (G1) que usó la técnica de lectura activa sobre conceptos básicos de Scrum y un grupo experimental 2 (G2) que participó en el juego SimulES basado en Scrum.

Este cuasiexperimento se realizó con el fin de (i) obtener evidencia preliminar acerca del uso de técnicas de aprendizaje activo en la enseñanza de conceptos básicos de Scrum, y (ii) verificar la hipótesis planteada (siendo H0 la hipótesis nula y H1 la hipótesis alternativa):

H0 (nula): No existe una diferencia significativa entre el conocimiento adquirido sobre Scrum por los participantes del grupo que jugaron SimulES (G2) y el conocimiento adquirido sobre Scrum por los participantes del grupo que utilizaron la técnica de lectura activa (G1).
H1 (alternativa): Existe una diferencia significativa entre el conocimiento sobre Scrum adquirido por los participantes del grupo que jugaron SimulES (G2) y el conocimiento sobre $S$ crum adquirido por los participantes del grupo que utilizaron la técnica de lectura activa (G1).

Diseñar este experimento involucró varias etapas. Dichas etapas se esquematizan en la Figura 2 y se detallan en los párrafos subsiguientes.

1) Selección de participantes: se convocó a los estudiantes del primer semestre del pregrado de Ingeniería de sistemas de la Universidad Eafit a través del correo electrónico y a través de anuncios en clase. Los estudiantes estaban matriculados en el curso de Principios de desarrollo de software, el cual introduce conceptos básicos relacionados con la Ingeniería de software.

Fuente: los autores.

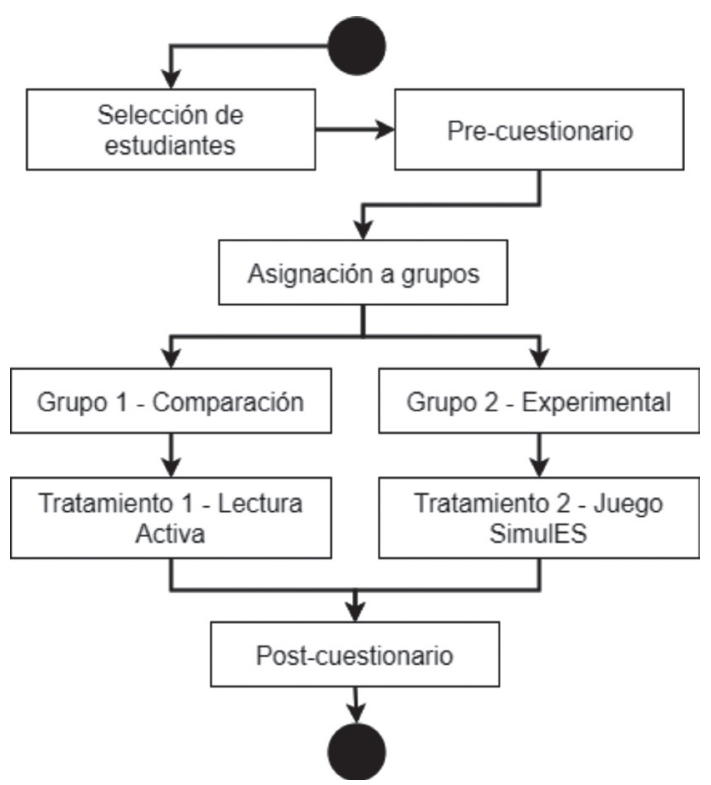

Figura 2. Etapas del cuasiexperimento con dos estrategias de aprendizaje activo para la enseñanza de Scrum.

2) Precuestionario: a la segunda etapa del cuasiexperimento asistieron 60 estudiantes, quienes resolvieron un precuestionario sobre su nivel de conocimientos en Scrum y la participación 
o no en cursos sobre este tema (ver Tabla 1). El precuestionario usado en este cuasiexperimento fue diseñado basado en la escala de Likert [35] y las preguntas incluían 5 opciones: (1) fuertemente en desacuerdo, (2) en desacuerdo, (3) ni de acuerdo ni en desacuerdo, (4) estoy de acuerdo, y (5) fuertemente de acuerdo. El precuestionario fue utilizado para llevar a cabo la conformación de grupos (que se describe en la siguiente etapa). Los resultados de este precuestionario son discutidos en la sección "Resultados y amenazas a la validez del cuasiexperimento".

Tabla 1. Preguntas del precuestionario.

P1. ¿Tengo conocimiento acerca de los conceptos
básicos de Scrum?
P2. ¿He participado en cursos (ya sean presenciales
o virtuales) sobre Scrum?

Fuente: los autores.

3) Asignación a grupos: utilizando como base los resultados del precuestionario anterior, se descartaron aquellos estudiantes que poseían conocimientos sobre Scrum. Además, se utilizó la información sobre el promedio acumulado de las notas de cada estudiante en el curso Principios de desarrollo de software, para finalmente conformar dos grupos, cada grupo con 18 integrantes.

Grupo 1 (G1), los integrantes de este grupo constituyen el grupo experimental 1 y usaron la lectura activa como estrategia de aprendizaje. Grupo 2 (G2), a los integrantes de este grupo se les proporcionó el juego SimulES y constituyen el grupo experimental 2.

4) Tratamientos: durante esta etapa se aplicaron dos técnicas de aprendizaje activo para la enseñanza de Scrum. Cada uno de los tratamientos duró 1 hora y 20 minutos.

Tratamiento A: este tratamiento se aplicó sólo a G1. Este grupo usó la técnica de lectura activa sobre conceptos básicos de Scrum.

Tratamiento B: este tratamiento se aplicó sólo a G2. Se dividió el grupo de 18 sujetos en 3 subgrupos ( 6 sujetos en cada uno) y cada subgrupo jugó con su copia del juego SimulES.

5) Post-cuestionarios: en esta etapa final del cuasiexperimento los estudiantes de ambos grupos respondieron a una serie de preguntas con el objetivo de valorar el conocimiento de los conceptos sobre Scrum y para conocer el grado de satisfacción generado por la actividad experimental (ver Tablas 2 y 3 ). Esta etapa duró 15 minutos. Los resultados de este postcuestionario son discutidos en la sección "Resultados y amenazas a la validez del cuasiexperimento".

\section{RESULTADOS Y AMENAZAS A LA VALIDEZ DEL CUASIEXPERIMENTO}

Los resultados obtenidos de la ejecución del cuasiexperimento se describen en el siguiente orden: 1) resultados para la asignación de grupos, es decir, del precuestionario; 2) resultados sobre conocimiento en Scrum, es decir, del post-cuestionario con las preguntas sobre Scrum; y 3) resultados sobre factores externos y satisfacción, es decir, del postcuestionario con las preguntas sobre factores externos y satisfacción con la experiencia de aprendizaje.

Además, para proporcionar relevancia estadística en el análisis de ambos cuestionarios, los resultados serán interpretados como se describe a continuación. Sea $H O$ la hipótesis nula y $H I$ la hipótesis alternativa; $G 1$ y $G 2$ los dos grupos experimentales usados para la comparación; y $p$ el estimador de probabilidad de rechazar erróneamente la hipótesis nula. Se definen entonces, las siguientes hipótesis alternativas: (i) $\mathrm{H} 1$ : $\mathrm{G} 2 \neq \mathrm{G} 1$, los resultados del grupo experimental 2 son diferentes a los del grupo experimental 1 , (ii) $\mathrm{H} 1$ : G2 < G1, los resultados en el grupo 2 son menores que los del grupo 1, o (iii) H1: G2 > G1, los resultados en el grupo 2 son mayores que los del grupo 1. Los resultados se compararon para cada respuesta de los cuestionarios, utilizando el

Tabla 2. Preguntas sobre factores externos y satisfacción con la experiencia.

P1. ¿El ambiente de trabajo de la experiencia fue intimidante?

P2. ¿Me sentí distraído por otros compañeros durante la experiencia?

P3. ¿Considero que el tiempo estipulado para el desarrollo de la experiencia fue el adecuado?

P4. ¿Considero que la experiencia fue divertida?

P5. ¿Considero que la experiencia propicia el aprendizaje y la adquisición de conocimientos? 
Tabla 3. Preguntas del post-cuestionario sobre el conocimiento de Scrum.

1. ¿Scrum sirve para gestionar equipos de desarrollo que quieren desarrollar un producto software?

2. ¿El Scrum master tiene un alto conocimiento del negocio y es quien cuida los intereses del cliente, en otras palabras, es el responsable por lograr el máximo valor empresarial para el proyecto?

3. El Scrum board permite de forma visual comprender y conocer el progreso de las tareas definidas a partir de cada historia de usuario (user story). Los cuatro tiempos o columnas mínimas de tiempo que se esperan ver en un Scrum board basado en Kanban son: ¿to do, doing, done, pruebas?

4. ¿El Product owner es quien gestiona el producto (y el retorno de la inversión), adicional a eso prioriza los requisitos o deseos establecidos por el cliente?

5. ¿Pueden ser parte del Scrum Team, desarrolladores, testers, analistas, arquitectos, redactores, diseñadores?

6. ¿Qué se puede entregar al final de un sprint por parte del equipo?

1. Incremento de software que funciona y está "Done".

2. Un conjunto de pruebas fallidas.

3. Un incremento de software con defectos conocidos en él.

4. Solo documentación.

7. Un sprint es:

1. Incremento de software que funciona y está "Done".

2. Un incremento de software con defectos conocidos en él.

3. Un intervalo prefijado durante el cual se crea un incremento de producto "Done".

8. Una historia de usuario es:

1. Descripción de una funcionalidad que debe incorporar un sistema de software y cuya implementación aporta valor al cliente.

2. Documentación.

3. Un intervalo prefijado durante el cual se crea un incremento de producto "Done".

test no paramétrico de Wilcoxon-Mann-Whitney [32], utilizando dos muestras independientes, con $n 1=18$ y $n 2=18$. Siendo $n 1$ y $n 2$ el número de integrantes de G1 y G2, respectivamente. El nivel de significación para todas las pruebas se estableció en $5 \%$, por lo que los valores de probabilidad de $p \leq 0,05$ se consideran significativos y $p \leq 0,01$ considerados altamente significativos. Lo cual significa que para todo valor de $p \leq 0,05$ se acepta $H 1$ y se rechaza $H O$.

\section{1) Resultados para la asignación de grupos}

Los resultados del precuestionario (ver Tabla 4) mostraron que no hay diferencia significativa entre $G 1$ y $G 2$ con respecto a sus conocimientos básicos en Scrum. Además, las respuestas del precuestionario confirman que no hay diferencias significativas con respecto a las preguntas $1(\mathrm{P} 1)$ y $2(\mathrm{P} 2),(\mathrm{P} 1 \mathrm{p}=1,000 ; \mathrm{P} 2 \mathrm{p}=0,163)$ entre ambos grupos. Las respuestas de $\mathrm{P} 1(\mathrm{G} 2 \overline{\mathrm{x}}=1,33$; G1 $\overline{\mathrm{x}}=1,33$ ) muestran que los estudiantes no tenían conocimientos de los conceptos básicos de Scrum; y las respuestas de $\mathrm{P} 2(\mathrm{G} 2 \overline{\mathrm{x}}=1,11 ; \mathrm{G} 1 \overline{\mathrm{x}}=1$, 00) demuestran que los estudiantes no habían participado en cursos de Scrum. Estos resultados eran una condición obligatoria para la validez del cuasiexperimento.

Tabla 4. Resumen de los resultados del precuestionario para G1 y G2 incluyendo los valores de la prueba no paramétrica Wilcoxon-Mann-Whitney con su nivel de significancia $\mathrm{p}$.

\begin{tabular}{|c|c|c|c|c|c|}
\hline \multirow{2}{*}{ Preguntas } & \multicolumn{2}{|c|}{ G2 } & \multicolumn{2}{|c|}{ G1 } & \multirow{2}{*}{ H1 p-value } \\
\hline & $\bar{x}$ & 0 & $\bar{x}$ & $\sigma$ & \\
\hline P1 & 1,33 & 0,49 & 1,33 & 0,49 & 1,000 \\
\hline P2 & 1,11 & 0,32 & 1,00 & 0,00 & 0,163 \\
\hline
\end{tabular}

\section{2) Resultados sobre conocimientos en Scrum}

Parte del post-cuestionario fue diseñado con la intención de medir el nivel de conocimiento adquirido sobre Scrum. Se diseñaron ocho preguntas, de las cuales las primeras cinco preguntas se debían responder seleccionando falso o verdadero, y en el caso de las últimas tres preguntas se debía seleccionar una opción verdadera entre tres opciones (ver Tabla 5). Con base en las respuestas a estas preguntas se pudo determinar cuánta información correcta acerca de los conceptos básicos de Scrum fue adquirida y retenida por parte de los estudiantes. En este caso, es más relevante realizar un análisis de los resultados del conjunto de respuestas, que de cada una de las respuestas a cada pregunta.

La Tabla 6 muestra que los integrantes de G1 (lectura activa) tuvieron un promedio de 7 respuestas correctas sobre un total de 8 preguntas; mientras que los integrantes de G2 (juego SimulES) tuvieron 
Tabla 5. Respuestas correctas a las preguntas de conocimientos básicos de Scrum.

\begin{tabular}{|l|c|c|c|c|c|c|c|c|}
\hline Pregunta & 1 & 2 & 3 & 4 & 5 & 6 & 7 & 8 \\
\hline Respuesta correcta & v & f & v & v & v & 1 & 3 & 1 \\
\hline
\end{tabular}

Tabla 6. Resumen de los resultados del postcuestionario (consolidado de respuestas correctas sobre conceptos básicos de Scrum) entre G1 y G2 incluyendo los valores de la prueba no paramétrica Wilcoxon-Mann-Whitney con su nivel de significancia $\mathrm{p}$.

\begin{tabular}{|c|c|c|c|}
\hline & G2 & G1 & \multirow{2}{*}{$\begin{array}{c}\text { H1 } \\
\text { p-value }\end{array}$} \\
\hline & $\bar{x}$ & $\bar{x}$ & \\
\hline $\begin{array}{l}\text { Consolidado } \\
\text { respuestas } \\
\text { correctas }\end{array}$ & $\begin{array}{ll}6,22 & 0,9\end{array}$ & $\begin{array}{lll}7,00 & 0,9\end{array}$ & $\neq \quad 0,019$ \\
\hline
\end{tabular}

un promedio de 6, 2 respuestas correctas sobre 8 . Aunque los resultados de ambos grupos parecen ser similares, el valor de p no supera el nivel de significancia, por tanto, hay evidencia para rechazar $\mathrm{H} 0$, es decir, con una confianza del 98\%; el nivel de conocimiento adquirido sobre Scrum es significativamente diferente entre los estudiantes que usaron la estrategia de lectura activa (G1) $(\overline{\mathrm{x}}=7,00)$ y los que jugaron el juego SimulES (G2) $(\overline{\mathrm{x}}=6,22), \mathrm{U}=91, \mathrm{Z}=-2,357, \mathrm{p} 1=0,019$, siendo $\mathrm{p} 1$ el p-valor con el que se prueba la hipótesis alternativa (i) $\mathrm{H} 1: \mathrm{G} 22 \neq \mathrm{G} 1$.

Se calcula el p-valor para probar la hipótesis alternativa (ii) $\mathrm{H} 1: \mathrm{G} 2<\mathrm{G} 1$, siendo $\mathrm{p} 2=0,010$, el valor de $\mathrm{p}$ no supera el nivel de significancia, por tanto, hay evidencia para rechazar $\mathrm{H} 0$, siendo mejores los resultados en el post-cuestionario para G1(lectura activa).

De igual manera se calcula el p-valor para probar la hipótesis (iii) H1: G2 > G1, siendo p3 = 0, 993, hay evidencia para rechazar $\mathrm{H} 1$.

\section{3) Resultados sobre factores externos y satisfacción}

El entorno y ambiente de trabajo del cuasiexperimento (factores externos) debe ser considerado adecuadamente, ya que puede representar una amenaza para la validez del experimento. En un salón de clases, se pueden presentar situaciones inesperadas, tales como: perturbaciones por parte de los participantes y el ruido percibido. Por lo tanto, se realizaron dos preguntas que tenían como objetivo descartar estas posibles amenazas al experimento (ver Tabla 2, preguntas 1 y 2). Los resultados de las preguntas sobre factores externos (ver Tabla 7) no muestran diferencias significativas ( $\mathrm{P} 1 \mathrm{p}=0,107$; $\mathrm{P} 2 \mathrm{p}=1,000)$ entre ambos grupos. Las respuestas de P1 (G2 $\overline{\mathrm{x}}=1,61$; G1 $\overline{\mathrm{x}}=2,11)$ muestran que los estudiantes no encontraron la experiencia intimidante; las respuestas de $\mathrm{P} 2(\mathrm{G} 2 \overline{\mathrm{x}}=2,39$; G1 $\overline{\mathrm{x}}=2,39$ ) muestran que los estudiantes se sintieron un poco distraídos. Los resultados de la pregunta P1 sirven para descartar amenazas a la validez del experimento con respecto a factores externos; sin embargo, aunque los resultados de la pregunta $\mathrm{P} 2$ se pueden ver como una amenaza a la validez del experimento, esta se mitiga ya que ese disturbio fue presenciado por ambos grupos.

Tabla 7. Resumen de los resultados del postcuestionario (preguntas factores externos) entre G1 y G2 incluyendo los valores de la prueba no paramétrica Wilcoxon-MannWhitney con su nivel de significancia p.

\begin{tabular}{|c|c|c|c|}
\hline \multirow{2}{*}{ Preguntas } & G2 & G1 & \multirow{2}{*}{$\begin{array}{c}\text { H1 } \\
\text { p-value }\end{array}$} \\
\hline & $\bar{x}$ & $\bar{x}$ & \\
\hline Pregunta 1 & $1,61 \quad 0,92$ & $2,11 \quad 1,02$ & 0,107 \\
\hline Pregunta 2 & $2,391,09$ & $2,391,09$ & 1,000 \\
\hline
\end{tabular}

Adicional a los factores externos, se definieron tres preguntas con el objetivo de obtener información subjetiva sobre la satisfacción que presentaron los alumnos al participar en cada una de las estrategias (ver Tabla 2, preguntas 3, 4 y 5). En este caso, el promedio de los resultados de cada respuesta de G2 fue más alto que los de G1 (ver Tabla 8). Incluso en una de las respuestas a las preguntas de satisfacción se ve una diferencia altamente significativa $(\mathrm{P} 3 \mathrm{p}=0$, 654; $\mathrm{P} 4 \mathrm{p}=0,003 ; \mathrm{P} 5 \mathrm{p}=0,097$ ). Las respuestas a P3 (G2 $\overline{\mathrm{x}}=4,06 ; \mathrm{G} 1 \overline{\mathrm{x}}=3,78 ; \mathrm{p}=0,654)$ muestran que los estudiantes encuentran adecuado el tiempo para desarrollar el experimento. Las respuestas a P4 (G2 $\bar{x}=4,39 ; \mathrm{G} 1 \overline{\mathrm{x}}=3,56 ; \mathrm{p}=0,003)$ muestran que los integrantes de G2 encontraron más divertida la experiencia que aquellos de G1. 
Las respuestas a P5 (G2 $\overline{\mathrm{x}}=4,28 ; G 1 \overline{\mathrm{x}}=3,89$; $\mathrm{p}=0,097)$ muestran que los estudiantes encuentran la experiencia propicia para el aprendizaje. Aunque ambos grupos mostraron resultados similares, los integrantes de G2 mostraron una diferencia significa en cuanto a la diversión, este último aspecto se podría explicar, ya que la diversión al participar de un juego puede ser más mayor que aquella en la que se participa de una lectura y discusión en clase.

Tabla 8. Resumen de los resultados del postcuestionario (preguntas satisfacción) entre G1 y G2 incluyendo los valores de la prueba no paramétrica Wilcoxon-Mann-Whitney con su nivel de significancia $\mathrm{p}$.

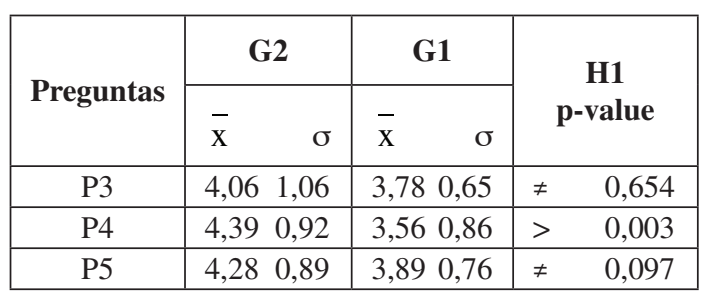

Dados los resultados anteriores podemos concluir que:

- No existen diferencias significativas entre los resultados de los grupos evaluados mediante el precuestionario; lo cual sirvió como base para la correcta formación de los grupos (ver Tabla 4).

- Se encontró una diferencia significativa en el resultado del "consolidado de respuestas correctas sobre conceptos básicos de Scrum" (ver Tabla 6), con el cual se evalúa el nivel de conocimiento adquirido sobre Scrum. El consolidado de respuestas correctas de G2 y de G1, muestra un valor de $\mathrm{p} 1=0,019$, y un p2 $=0,010$, siendo mejores los resultados para G1 (estrategia de lectura activa).

- No existen diferencias significativas entre los resultados de los grupos evaluados mediante el post-cuestionario de preguntas de factores externos (ver Tabla 7).

- Se encontró una diferencia significativa en el resultado de $\mathrm{P} 4$ de los grupos evaluados mediante el post-cuestionario (preguntas de satisfacción; ver Tabla 8), siendo mejor el resultado para G2. Para las otras dos respuestas (P3 y P5) no hubo diferencias significativas.

\section{CONCLUSIONES Y TRABAJO FUTURO}

El estudio tenía como objetivo principal comparar dos técnicas de aprendizaje activo buscando una apropiación del conocimiento de los estudiantes sobre el marco de trabajo ágil Scrum. Para ello se usó un cuasiexperimento con el fin de comparar la efectividad de las técnicas en términos de la adquisición de conocimiento.

Ambas estrategias probadas en esta investigación mejoraron el nivel de conocimiento sobre Scrum de manera significativa. Sin embargo, en promedio, el nivel de conocimiento adquirido sobre los conceptos de Scrum después de que los estudiantes usaron la estrategia de lectura activa $(M=7,00)$ fue significativamente más alto que los del grupo que jugó con SimulES $(M=6,22), U=91, Z=-2,357$, $\mathrm{p}<0,05$.

Por tanto, la estrategia de lectura activa parece tener un mayor efecto en el nivel de conocimiento adquirido sobre Scrum en comparación con la estrategia del juego SimulES.

Por otro lado, los resultados de la encuesta de satisfacción mostraron que los participantes que jugaron SimulES encontraron más divertida la experiencia que aquellos que leyeron de manera activa y además los estudiantes encontraron la experiencia propicia para el aprendizaje.

Dado los múltiples estudios comparativos entre estrategias de aprendizaje activo y aprendizaje tradicional y teniendo en cuenta los resultados arrojados por dichos estudios que sugieren que el aprendizaje es mejorado usando las estrategias pedagógicas activas, es razonable plantearse inquietudes sobre qué tipo de aprendizaje activo es el más apropiado y eficaz para ciertos temas o tipos de estudiantes. De ahí la relevancia de este trabajo que aporta resultados en ese sentido.

Teniendo en cuenta dichos resultados, es razonable pensar que ambas estrategias tienen sus fortalezas y debilidades y, por lo tanto, combinarlas sería una buena opción para obtener los beneficios de cada una. La adecuada selección de las estrategias de enseñanza es de vital importancia para la buena formación de los futuros ingenieros de software. De modo que para los profesores es importante conocer 
diversas estrategias y las ventajas y limitaciones que estas aportan en los procesos de aprendizaje de los estudiantes para que adapten los procesos de enseñanza en consecuencia.

El trabajo desarrollado tiene como limitante el tamaño de la muestra y la ubicación, pues con una muestra pequeña y en un solo lugar, los resultados de este estudio no pueden ser generalizados. Igualmente, la imposibilidad de hacer una selección aleatoria de la muestra es también una amenaza para la generalización de los resultados de este estudio.

Es necesario realizar estudios futuros para investigar la mejor manera de integrar ambos enfoques y explorarlos bajo diferentes resultados de aprendizaje. Otro trabajo futuro podría ser experimentar con diferentes estrategias de enseñanza para cada estudiante, con el fin de caracterizar los perfiles de los estudiantes y asociarlos a las estrategias que les han permitido obtener mejores resultados.

\section{REFERENCIAS}

[1] M. Prince. "Does active learning work? A review of the research." Journal of engineering education, Vol. $93 \mathrm{~N}^{\circ} 3$, pp. 223-231. 2004.

[2] S. Freeman, S. Eddya, M. McDonougha, M. Smithb, N. Okoroafora, H. Jordta, and M. Pat Wenderotha. "Active learning increases student performance in science, engineering, and mathematics". Vol. 111, No 23, pp. 84108415. 2014.

[3] Universidad EAFIT. “¿Qué es el aprendizaje activo?". 10/04/2017. Fecha de consulta: 19/08/2017. URL: http://www.eafit.edu.co/ proyecto50/aprendizaje/aprendizajeactivo/ Paginas/que-es-el-aprendizaje-activo.aspx

[4] J.E. Froyd and S.S. Effects. "Evidence for the Efficacy of Student-active Learning Pedagogies Cooperative and Small-group Learning Peda gogies", Vol. 66, $\mathrm{N}^{\circ} 1$, pp. 64-74. 2007.

[5] B.S. Bloom. "Taxonomía de los objetivos de la educación: la clasificación de las metas educacionales, manuales I y II'. El Ateneo. Edición 6. Buenos Aires, Argentina. pp. 257 páginas. 1981.

[6] A. King. "From sage on the stage to guide on the side". College teaching, Vol. $41 \mathrm{~N}^{\mathrm{o}} 1$, pp. 30-35. 1993.
[7] R.E. Mayer. "Aids to prose comprehension", Educational Psychologist, Vol. 19, pp. 30-42. 2009.

[8] P.C. Wankat and F.S. Oreovicz. "Psychological type and learning". Retrieved 30 (1993): 2012.

[9] I.B. Myers and M.H. McCaulley. "Manual: A guide to the development and use of the Myers-Briggs". Type Indicator, Consulting Psychologists Press. Palo Alto, CA. 1985.

[10] C.G. Jung. "Psychological Types". Princeton University Press. Princeton, NJ. 1971. (originally published in 1921).

[11] B. Oakley, R. Brent, R.M. Felder and I. Elhajj. "Turning student groups into effective teams". Journal of student centered learning. Vol. $2 \mathrm{~N}^{\circ}$ 1, pp. 9-34. 2004.

[12] J.A. Van Amburgh, J.W. Devlin, J.L. Kirwin and D.M. Qualters. "A Tool for Measuring Active Learning in the Classroom". Am J Pharm Educ. 2007.

[13] What is Active Reading? - Definition \& Strategies. 19/08/2017. URL: http://study. com/academy/lesson/what-is-active-readingdefinition-strategies.html

[14] H.D. Benington. "Production of Large Computer Programs". IEEE Annals of the History of Computing. Vol. 5, pp. 350-361. 1983. DOI:10.1109/MAHC.1983.10102.

[15] W. Royce. "Managing the Development of Large Software Systems". IEEE WESCON. Los Angeles, USA. 1970.

[16] T.E. Bell and T.A. Thayer. "Software requirements: Are they really a problem?". 2nd international conference on Software engineering. IEEE Computer Society Press. 1976.

[17] B. Boehm. "A Spiral Model of Software Development and Enhancement", ACM SIGSOFT Software Engineering Notes. Vol. 11, No 4, pp. 14-24. 1986.

[18] B.W. Boehm and R. Beach. "Guidelines for Verifying and Validating Software Requirements and Design Specifications". Euro IFIP 79. Londres. 1979.

[19] G.J. Myers. "The Art of Software Testing". John Wiley \& Sons. Estados Unidos. 1979. ISBN: 0.471-04328-1.

[20] E.S.F. Cardozo, J.B. Neto, A. Barza, C. França and F.Q.B. da Silva. "SCRUM and Productivity in Software Projects: Systematic 
Literature Review". Proceedings of the 14th International Conference on Evaluation and Assessment in Software Engineering, pp. 131-134. 2010.

[21] M. Mahalaskshmi and M. Sundararajan. "Traditional SDLC Vs Scrum Methodology A Comparative Study". International Journal of Emerging Technology and Advanced Engineering, Vol. $3 \mathrm{~N}^{\circ}$ 6, pp. 192-196. 2013.

[22] V. Mahnic. "Scrum in software engineering courses: an outline of the literature". Proceedings of the 47th ACM Technical Symposium on Computing Science Education, pp. 319-320. 2016.

[23] K. Schwaber and J. Sutherland. "The Scrum Guide", 22/06/2015, 19/08/2017. URL: http:// www.scrumguides.org/.

[24] M. Paasivaara, V. Heikkilä, C. Lassenius and T. Toivola. "Teaching students Scrum using LEGO blocks". Companion Proc. 36th Inter. Conf. on Software Engng., pp. 382-391. Hyderabad, India. 2014.

[25] J.M. Fernandes and S.M. Sousa. "PlayScrum - A card game to learn the scrum agile method". 2nd Int. Conf. Games Virtual Worlds Serious Appl. VS-GAMES 2010, pp. 52-59. 2010.

[26] E.M. Figueiredo, C.A. Lobato, K.L. Dias, J.C. Leite and C.J.P. Lucena. "Um Jogo para o Ensino de Engenharia de Software Centrado na Perspectiva de Evolução". Workshop sobre Educação em Computação (WEI - 2007), Rio de Janeiro. 2007.

[27] A. Baker, E. Navarro and A. van der Hoek. "Problems and Programmers: an educational software engineering card game". Proceedings 25th Intern. Conference on Software Engineering, IEEE Computer Society Press, Portland. 2003.

[28] E.S. Monsalve, P. Vallejo, R. Mazo, and D. Correa. "Transparency as a learning strategy to teach Software Engineering Using SimulES to teach Software Engineering transparently", presentado en 12th Colombian Conference on Computing http://www.uao.edu.co/12ccc/ en/pp. 1-8. 2017.
[29] E.S. Monsalve, V.M.B. Werneck and J.C.S.D.P. Leite. "Teaching Software Engineering with SimulES-W". XXIV Conference on Software Engineering Education and Training (CSEE\&T). Hawaii. 2011.

[30] E.S. Monsalve, J.C.S.D.P. Leite and V.M.B. Werneck. "Transparently Teaching in the Context of Game-based Learning: The Case of SimulES-W'. International Conference on Software Engineering, 2, art. $\mathrm{N}^{\mathrm{o}} 7202984$, pp. 343-352. 2015. ISBN: 978-147991934-5. DOI: 10.1109/ICSE.2015.167.

[31] E.S. Monsalve, V. Werneck and J.C.S.P. Leite. "SimulES-W: Um Jogo para o Ensino de Engenharia de Software". III Fórum de Educação em Engenharia de Software. Salvador, Bahia, BA. 2010.

[32] J.C. Carver, L. Jaccheri, S. Morasca and F. Shull. "A checklist for integrating student empirical studies with research and teaching goals". Empirical Software Engineering, Vol. $15 \mathrm{~N}^{\circ}$ 1, pp. 35-59. 2010.

[33] V.B. Kampenes, T. Dybå, J.E. Hannay and D.I. Sjøberg. "A systematic review of quasi-experiments in software engineering". Information and Software Technology, Vol. 51 $\mathrm{N}^{\circ}$ 1, pp. 71-82. 2009.

[34] J, Münch and K, Schmid. "Perspectives on the Future of Software Engineering". Springer-Verlag Berlin Heidelberg. pp. 258. 2013. ISBN 978-3-642-37395-4.

[35] N. Flores. "Patterns and Tools for Improving Framework Understanding: a Collaborative Approach". Doctoral dissertation, University of Porto. 2012.

[36] D. Vlachopoulos and A. Makri, "The effect of games and simulations on higher education: a systematic literature review", Vol. 14, $\mathrm{N}^{\mathrm{o}} 1.2017$.

[37] L.M. Cleveland, J.T. Olimpo, and S.E. DeChenne-Peters, "Investigating the relationship between instructors' use of activelearning strategies and students' conceptual understanding and affective changes in introductory biology: A comparison of two active-learning environments". CBE Life Sci. Educ., Vol. 16, N 2. 2017. 